\title{
A phase lb study of utomilumab (PF-05082566) in combination with mogamulizumab in patients with advanced solid tumors
}

Ezra E. W. Cohen ${ }^{1 *}$, Michael J. Pishvaian², Dale R. Shepard ${ }^{3}$, Ding Wang ${ }^{4}$, Jared Weiss ${ }^{5}$, Melissa L. Johnson $^{6}$, Christine H. Chung ${ }^{7}$, Ying Chen ${ }^{8}$, Bo Huang ${ }^{9}$, Craig B. Davis ${ }^{8}$, Francesca Toffalorio ${ }^{10}$, Aron Thall ${ }^{8}$ and Steven F. Powell ${ }^{11}$

\begin{abstract}
Background: Expressed on activated T and natural killer cells, 4-1BB/CD137 is a costimulatory receptor that signals a series of events resulting in cytokine secretion and enhanced effector function. Targeting 4-1BB/CD137 with agonist antibodies has been associated with tumor reduction and antitumor immunity. C-C chemokine receptor 4 (CCR4) is highly expressed in various solid tumor indications and associated with poor prognosis. This phase lb, open-label study in patients with advanced solid tumors assessed the safety, efficacy, pharmacokinetics, and pharmacodynamics of utomilumab (PF-05082566), a human monoclonal antibody (mAb) agonist of the T-cell costimulatory receptor 4-1BB/CD137, in combination with mogamulizumab, a humanized mAb targeting CCR4 reported to deplete subsets of regulatory $T$ cells (Tregs).

Methods: Utomilumab $1.2-5 \mathrm{mg} / \mathrm{kg}$ or $100 \mathrm{mg}$ flat dose every 4 weeks plus mogamulizumab $1 \mathrm{mg} / \mathrm{kg}$ (weekly in Cycle 1 followed by biweekly in Cycles $\geq 2$ ) was administered intravenously to 24 adults with solid tumors. Blood was collected pre- and post-dose for assessment of drug pharmacokinetics, immunogenicity, and pharmacodynamic markers. Baseline tumor biopsies from a subset of patients were also analyzed for the presence of programmed cell death-ligand 1 (PD-L1), CD8, FoxP3, and 4-1BB/CD137. Radiologic tumor assessments were conducted at baseline and on treatment every 8 weeks.
\end{abstract}

Results: No dose-limiting toxicities occurred and the maximum tolerated dose was determined to be at least $2.4 \mathrm{mg} / \mathrm{kg}$ per the time-to-event continual reassessment method. No serious adverse events related to either treatment were observed; anemia was the only grade 3 non-serious adverse event related to both treatments. Utomilumab systemic exposure appeared to increase with dose. One patient with PD-L1-refractory squamous lung cancer achieved a best overall response of partial response and 9 patients had a best overall response of stable disease. No patients achieved complete response. Objective response rate was 4.2\% (95\% confidence interval: 0.1-21.1\%) per RECIST 1.1. Depletion of Tregs in peripheral blood was accompanied by evidence of Tcell expansion as assessed by T-cell receptor sequence analysis.

Conclusions: The combination of utomilumab/mogamulizumab was safe and tolerable, and may be suitable for evaluation in settings where CCR4-expressing Tregs are suppressing anticancer immunity.

Trial registration: ClinicalTrials.gov identifier: NCT02444793.

Keywords: 4-1BB, CD137, Utomilumab, Mogamulizumab, Solid tumors

\footnotetext{
*Correspondence: ecohen@ucsd.edu

'UC San Diego Health, Moores Cancer Center, University of California San

Diego, 3855 Health Sciences Drive, La Jolla, CA 92093, USA

Full list of author information is available at the end of the article
}

(c) The Author(s). 2019 Open Access This article is distributed under the terms of the Creative Commons Attribution 4.0 International License (http://creativecommons.org/licenses/by/4.0/), which permits unrestricted use, distribution, and reproduction in any medium, provided you give appropriate credit to the original author(s) and the source, provide a link to the Creative Commons license, and indicate if changes were made. The Creative Commons Public Domain Dedication waiver (http://creativecommons.org/publicdomain/zero/1.0/) applies to the data made available in this article, unless otherwise stated. 


\section{Background}

Cancer immunotherapy, in particular monoclonal antibody $(\mathrm{mAb})$ antagonists of the programmed cell death protein 1 (PD-1)/programmed cell death-ligand 1 (PDL1) pathway, has substantially helped patients with a variety of solid tumor types, including non-small-cell lung cancer (NSCLC) [1], squamous cell carcinoma of the head and neck (SCCHN) [2], melanoma [3], bladder cancer [4], and renal cell carcinoma [5]. Patients whose tumors do not respond to PD-1/PD-L1 antagonists represent an increasingly recognized area of unmet need [6]. Tumor cell extrinsic mechanisms, such as the lack of $\mathrm{T}$ cells or the presence of immunosuppression [7], may define a subclass of patients who would benefit from combinations that provide costimulatory signals to antitumor $\mathrm{T}$ cells while removing immunosuppressive cells. One such combination is utomilumab plus mogamulizumab.

Utomilumab (PF-05082566) is a fully human immunoglobulin G2 agonist mAb that binds to human 4-1BB/ CD137 with high affinity and specificity [8]. 4-1BB/ CD137 is a costimulatory receptor of the tumor necrosis factor receptor superfamily expressed on activated immune cells, including $\mathrm{T}$ cells [9], dendritic cells [10], and natural killer cells [11]. 4-1BB/CD137 agonists promote immune cell proliferation, survival, cytokine production [12-15], formation of immunologic memory, and sustained T-cell immune responses [16-18]. Lymphocyte activation and favorable antitumor responses have been elicited by utomilumab as well as other 4-1BB/CD137 agonists in multiple preclinical models [8, 13, 19-21]. A phase I trial of utomilumab recently reported a favorable safety profile and preliminary antitumor activity [22].

Mogamulizumab is a recombinant humanized $\mathrm{mAb}$ targeting C-C chemokine receptor 4 (CCR4, CD194). It was first approved in Japan in 2012 for relapsed or refractory CCR4 ${ }^{+}$adult T-cell leukemia-lymphoma (ATL), and approval for first-line treatment of CCR4 ${ }^{+}$ATL was granted in 2014. Approval for additional indications of relapsed or refractory $\mathrm{CCR}_{4}^{+}$peripheral $\mathrm{T}$-cell lymphoma and cutaneous T-cell lymphoma was gained in 2014. In 2018 it was approved by the US Food and Drug Administration and European Medicines Agency for the treatment of relapsed or refractory mycosis fungoides or Sézary syndrome after at least 1 prior systemic therapy. CCR4 has been observed on regulatory $\mathrm{T}$ cells (Tregs) [23]. In vitro or in vivo mogamulizumab treatment selectively depleted $\mathrm{CCR}^{+}$Tregs and is associated with increased levels of tumorantigen-specific $\mathrm{T}$ cells $[24,25]$.

The combination hypothesis has been evaluated by in vivo experiments in a murine melanoma model in which the antitumor activity of 4-1BB/CD137 was significantly improved when given in combination with an
anti-CD4 $\mathrm{mAb}$ that depleted Tregs as well as other $\mathrm{CD} 4^{+}$cells [26]. Anti-4-1BB/CD137 treatment resulted in the polyclonal expansion and differentiation of antitumor $\mathrm{CD}^{+} \mathrm{T}$ cells into effective antitumor agents, whereas $\mathrm{CD} 4^{+} \mathrm{T}$-cell depletion facilitated the infiltration of immune cells into the tumors and removed Treg hindrance [26].

The mechanistic data for utomilumab and mogamulizumab as single agents coupled with the preclinical outcomes supported clinical evaluation of the hypothesis that depletion of $\mathrm{CCR}^{+}$Tregs by mogamulizumab would enhance the efficacy of antitumor immune responses expanded by utomilumab.

This phase Ib study investigated safety, efficacy, pharmacokinetics (PK), and pharmacodynamics of utomilumab plus mogamulizumab in patients with advanced solid tumors previously unresponsive to currently available therapies or for whom no standard therapy was available.

\section{Patients and methods \\ Study design and objectives}

This phase I, open-label, multicenter, multiple-dose study was approved by the institutional review boards at all nine centers in the US. Patients were enrolled between May 26, 2015 and February 7, 2017 (study completion October 10, 2017). The study was conducted in compliance with the ethical principles originating in or derived from the Declaration of Helsinki and in compliance with the International Council for Harmonization Good Clinical Practice Guidelines. All patients provided written informed consent. The study is registered on ClinicalTrials.gov (NCT02444793).

The primary objective of the study was to estimate the maximum tolerated dose (MTD) of utomilumab in combination with mogamulizumab in patients with advanced solid tumors. Secondary objectives included assessment of the safety profile, PK, immunogenicity, and antitumor activity of the combination. Exploratory objectives included the pharmacodynamic effect on immune parameters in blood.

\section{Patients}

Refractory patients had a previously documented best overall response (BOR) of non-complete response $(\mathrm{CR}) /$ partial response $(\mathrm{PR}) /$ stable disease $(\mathrm{SD})$ on $\mathrm{PD}$ 1/PD-L1 treatment (includes progressive disease and clinical deterioration); relapsed patients had documented $B O R$ of $C R / P R / S D$ but later progressed on PD-1/PD-L1 treatment (includes progressive disease and clinical deterioration).

Patients were not eligible if they had a history of autoimmune disease; systemic anticancer therapy within 28 days prior to registration; radiation therapy within 14 
days prior to registration; therapeutic or experimental mAbs within 28 days prior to registration; active and clinically significant bacterial, fungal, or viral infection; live vaccine within 30 days prior to registration; or systemic corticosteroid therapy or any other form of immunosuppressive therapy within 14 days prior to registration.

\section{Treatment}

The starting dose for intravenous utomilumab was 1.2 $\mathrm{mg} / \mathrm{kg}$ every 4 weeks, with escalation to $2.4 \mathrm{mg} / \mathrm{kg}$ and 5 $\mathrm{mg} / \mathrm{kg}$ in the subsequent cohorts following the time-toevent continual reassessment method (TITE-CRM). A flat dosing of $100 \mathrm{mg}$ utomilumab was also assessed. Intravenous mogamulizumab $1 \mathrm{mg} / \mathrm{kg}$ was administered weekly for 4 consecutive weeks and biweekly thereafter, following utomilumab dosing. Treatment with study drugs was to continue until the first occurrence of one of the following: completion of 24 months of treatment, disease progression, patient refusal to continue, unacceptable toxicity, or study termination by the sponsor.

\section{Study assessments}

Safety

Safety assessments included dose-limiting toxicities (DLTs) in the first 2 cycles and adverse events (AEs) characterized by type, frequency, severity (as graded by $\mathrm{Na}$ tional Cancer Institute Common Terminology Criteria for Adverse Events version 4.03). Causality was first assigned by the site Principal Investigator and then all serious AEs (SAEs) were adjudicated at a regular conference involving all sites and sponsor. The following AEs were considered DLTs if they were attributable to one or both study drugs: grade 4 neutropenia, febrile neutropenia, grade $\geq 3$ neutropenic infection, grade $\geq 3$ thrombocytopenia with bleeding, grade 4 thrombocytopenia, grade $\geq 3$ nonhematologic abnormalities, and grade 4 aminotransferase/ alanine aminotransferase increase. The MTD was defined as the highest combination dose with a DLT rate $<30 \%$ from the TITE-CRM model estimate.

\section{PK and immunogenicity}

Blood for utomilumab PK assessment was collected at predose and end of utomilumab infusion on Day 1 of Cycles 1-4; on Day 1 at predose and end of utomilumab infusion; at 2, 6, and $168 \mathrm{~h}$ (Day 8) and $336 \mathrm{~h}$ (Day 15) after the start of utomilumab infusion in Cycle 5; predose on Day 1 of Cycles 8,12, 16, 20, and 24; and end of treatment (EOT). PK samples for mogamulizumab were collected at predose and end of mogamulizumab infusion on Days 1, 8, 15, and 22 of Cycle 1; predose of Cycles 2-4; on Day 1 at predose and at the end of mogamulizumab infusion, and at 6 and $168 \mathrm{~h}$ after the start of the mogamulizumab infusion, and predose on
Day 15 of Cycle 5; predose on Day 1 of Cycles 8, 12, 16, 20, and 24; and EOT. Samples were assayed using validated enzyme-linked immunosorbent assays in compliance with standard operating procedures of the study sponsor (Pfizer, New York, NY, USA) for utomilumab and of Kyowa Hakko Kirin (KHK; Tokyo, Japan) for mogamulizumab. Standard serum PK parameters were estimated for both drugs using non-compartmental analysis.

Blood samples for antidrug antibody (ADA) assessments were collected at predose on Day 1 of Cycles 1, 3, $5,8,12,16,20$, and 24, and EOT. If ADAs were detected, additional samples were collected approximately every 8 weeks until ADA levels returned to baseline. Serum samples were assayed for ADAs using a validated electrochemiluminescence (ECL) assay (anti-utomilumab) and ECL-based ligand-binding assay (anti-mogamulizumab) in compliance with standard operating procedures of the sponsor (anti-utomilumab) and KHK (anti-mogamulizumab). ADA-positive samples were further tested for neutralizing antibodies (NAb) using a validated cell-based luciferase assay (anti-utomilumab) or ECL-based ligand-binding assay (anti-mogamulizumab).

\section{Pharmacodynamic assessments}

Readouts included changes in peripheral blood biomarkers, including cytokines, distribution of lymphocyte subpopulations, and frequency of T-cell receptor (TCR) sequences. Blood was collected for immunomodulation/ cytokine-release biomarkers at pre-infusion of utomilumab on Day 1 and at the end of mogamulizumab infusion for Cycles 1-4; pre-infusion of utomilumab on Day 1 , at the end of utomilumab infusion, and 2 and $6 \mathrm{~h}$ after the start of the utomilumab infusion of Cycle 5. Blood for the characterization of lymphocyte subpopulations was collected at predose on Day 1, and 2, 6, and $168 \mathrm{~h}$ (Day 8) and 336 h (Day 15) after the start of infusion of Cycles 1 and 5.

Analysis of serum cytokines and lymphocyte subpopulations in peripheral blood was performed as described by Tolcher et al. [27]. Lymphocyte subpopulations in the current report were defined using CD45, CD3, CD4, CD8, CD25, CD127, CD45RA, and CCR7. Expanded TCR sequences were quantified as described by Rytlewski et al. [28]. Statistical assessments of effects seen in the flow cytometry and TCR sequence expansion analyses were performed using Wilcoxon signed-rank testing and Wilcoxon rank-sum testing, respectively.

\section{Characterization of baseline tumor biopsies}

Immunohistochemistry was performed to detect the presence of PD-L1, CD8, FoxP3, and 4-1BB/CD137 in the whole tumor and invasive margin (IM) of pretreatment tumor biopsies. Immunohistochemistry testing of 
PD-L1 (clone E1L3N; Cell Signaling, Danvers, MA), CD8 (clone C8/144B; Dako, Carpinteria, CA), FoxP3 (clone 236A/E7; Cell Signaling), and 4-1BB/CD137 (BBK-2; ThermoFisher, Rockford, IL) was performed by Mosaic Laboratories, LLC (Lake Forest, CA).

\section{Antitumor activity}

Radiologic tumor assessments were conducted at baseline within 28 days prior to treatment, and on treatment every 8 weeks, starting from Cycle 1 Day 1 (up to 1 year), then every 3 months. Assessments were also to be conducted whenever disease progression was suspected, at EOT, and during follow-up visits. Response was assessed using RECIST1.1. Objective response was defined as BOR of CR or PR from the date of first dose of study treatment until disease progression. Both $\mathrm{CR}$ and $\mathrm{PR}$ were confirmed by repeat assessments performed no fewer than 4 weeks after the criteria for response were first met.

\section{Statistical analyses}

A modified TITE-CRM method with cyclical adaptive weight function was applied [29, 30]. The MTD was estimated as the highest dose level associated with a $<30 \%$ estimated DLT rate per the modified TITE-CRM design. A dose-escalation steering committee was established to facilitate the trial conduct process [31]. A sample size of 30 was estimated to provide an accurate estimate of the MTD and to detect any unexpected toxicity occurring at $5 \%$ rate (in a non-dose-dependent fashion), with a probability of 0.79 , and occurring at $10 \%$ rate with a probability of 0.96 . The objective response was summarized with objective response rate (ORR), and exact 2-sided 95\% confidence interval (CI) for ORR was calculated using the Clopper-Pearson method. Time-to-event endpoints were analyzed using the Kaplan-Meier method. Point estimates of Kaplan-Meier rates and median times were presented with their 95\% CIs. The CIs for the median were calculated according to the Brookmeyer and Crowley method.

\section{Results}

\section{Patients and treatment}

In all, 24 patients received mogamulizumab $1 \mathrm{mg} / \mathrm{kg}$ in combination with utomilumab dosed as follows: $1.2 \mathrm{mg} /$ $\mathrm{kg}(n=11), 2.4 \mathrm{mg} / \mathrm{kg}(n=4), 5 \mathrm{mg} / \mathrm{kg}(n=3)$, and 100 $\mathrm{mg}$ flat dose $(n=6)$. Most patients were male $(79.2 \%)$ and white (79.2\%). The mean (range) age was 63.9 (53$75)$ years. There were 11 patients with SCCHN, 10 with $\operatorname{NSCLC~(~} n=7$ squamous and 3 adenocarcinoma), 2 patients with colorectal cancer, and 1 patient with ovarian cancer, assessed by Response Evaluation Criteria in Solid Tumors (RECIST 1.1). The majority (91.7\%) of patients had received at least 2 lines of anticancer drug therapy
(Table 1). Median (range) duration of treatment was 16 (4.0-41.3) weeks. All of the patients with squamous $\operatorname{NSCLC}(n=7), 1$ with lung adenocarcinoma, and 7 with SCCHN were relapsed or refractory to anti-PD-1/PD-L1 checkpoint inhibitor therapy. Nine (37.5\%) and 15 (62.5\%) patients had baseline Eastern Cooperative Oncology Group performance status 0 and 1, respectively. The dose-expansion phase of the study was not initiated due to marginal efficacy.

\section{Safety}

No DLTs were observed at any utomilumab dose $(1.2 \mathrm{mg} /$ $\mathrm{kg}, 2.4 \mathrm{mg} / \mathrm{kg}, 5 \mathrm{mg} / \mathrm{kg}, 100 \mathrm{mg}$ flat dose) in combination with mogamulizumab $1 \mathrm{mg} / \mathrm{kg}$. Although no DLTs were observed up to $5 \mathrm{mg} / \mathrm{kg}$, the estimated recommended Phase II dose was at least $2.4 \mathrm{mg} / \mathrm{kg}$ per the TITE-CRM method; as the $5 \mathrm{mg} / \mathrm{kg}$ cohort only enrolled 3 patients, this dose was not fully explored in this respect. The most common (in $\geq 25 \%$ of patients), all-causality AEs were fatigue (45.8\%), rash $(29.2 \%)$, and diarrhea $(25.0 \%)$, all of grade 1 or grade 2 severity. Eight (33.3\%) patients experienced all-causality grade 3-4 AEs. Ten (41.7\%) patients experienced serious AEs (SAEs), all determined to be unrelated to utomilumab or mogamulizumab; AE causality was initially assessed by the site Principal Investigator and all SAEs were adjudicated during regular conferences involving all sites and sponsor. The majority of the treatment-related AEs were grade 1 or 2 , and none were grade 4 or 5 . Two $(8.3 \%)$ patients in the utomilumab $100 \mathrm{mg} /$ mogamulizumab $1 \mathrm{mg} / \mathrm{kg}$ treatment group experienced three grade 3 AEs determined to be related to treatment: pneumonitis (utomilumab-related), hypophosphatemia (mogamulizumab-related), and anemia (both treatments). Three (12.5\%) patients experienced grade $5 \mathrm{AEs}$, determined to be unrelated to either treatment. Of these patients, 2 occurred within 30 days after the last dose of study treatment and were due to malignant neoplasm progression/disease progression. The third patient died due to sepsis during the follow-up period (within 60 days after the last dose of study treatment).

\section{PK and immunogenicity}

Five patients had sufficient data to calculate PK parameters at Cycle 5. Utomilumab systemic exposure based on area under the serum concentration-time curve to last measureable dose and maximum serum concentration values appeared to increase with increasing dose. Due to the low number of patients, the relationship between serum PK parameters and dose could not be fully determined (Table 2).

Following co-administration with utomilumab, mogamulizumab PK was similar across groups, with accumulation observed following multiple-dose administration (Additional file 1 and Additional file 2). 
Table 1 Primary diagnosis and prior anti-cancer treatment

\begin{tabular}{|c|c|c|c|c|c|}
\hline \multirow{2}{*}{$\begin{array}{l}\text { Number (\%) of } \\
\text { patients }\end{array}$} & \multicolumn{5}{|c|}{ Mogamulizumab 1 mg/kg + Utomilumab, by Dose Group } \\
\hline & $\begin{array}{l}1.2 \mathrm{mg} / \mathrm{kg} \\
\mathrm{n}=11\end{array}$ & $\begin{array}{l}100 \mathrm{mg} \\
n=6\end{array}$ & $\begin{array}{l}2.4 \mathrm{mg} / \mathrm{kg} \\
n=4\end{array}$ & $\begin{array}{l}5 \mathrm{mg} / \mathrm{kg} \\
n=3\end{array}$ & $\begin{array}{l}\text { Total } \\
n=24\end{array}$ \\
\hline CRC & 1 & 0 & 1 & 0 & 2 \\
\hline NSCLC & 2 & 6 & 1 & 1 & 10 \\
\hline Ovarian Cancer & 0 & 0 & 0 & 1 & 1 \\
\hline SCCHN & 8 & 0 & 2 & 1 & 11 \\
\hline \multicolumn{6}{|c|}{ Prior anti-cancer drug regimens } \\
\hline 1 & $1(9.1)$ & 0 & $1(25.0)$ & 0 & $2(8.3)$ \\
\hline 2 & $3(27.3)$ & $3(50.0)$ & 0 & 0 & $6(25.0)$ \\
\hline 3 & $2(18.2)$ & $2(33.3)$ & $1(25.0)$ & $3(100.0)$ & $8(33.3)$ \\
\hline$\geq 4$ & $5(45.5)$ & $1(16.7)$ & $2(50.0)$ & 0 & $8(33.3)$ \\
\hline
\end{tabular}

CRC Colorectal cancer, NSCLC Non-small-cell lung cancer, SCCHN Squamous cell cancer of head and neck

Thirteen of $24(54.2 \%)$ patients that received utomilumab tested positive for treatment-induced ADA and 11 of 24 (45.8\%) were positive for NAb. One (4.2\%) patient who received mogamulizumab was confirmed positive for treatment-induced ADA; no one in this group tested positive for NAb. The median onset for treatment-induced ADA against utomilumab was 56.01 days (interquartile range [Q1, Q3]: 55.88, 56.13) and the median onset for NAb was 56.13 days (interquartile range [Q1, Q3]: 55.96, 56.97). The median duration of ADA and NAb was 0 and 0 days, respectively (interquartile range [Q1, Q3]: 0, 55.95 for ADA and 0, 62.84 for NAb). There was no substantial impact of ADA/NAb on PK and safety when utomilumab and mogamulizumab were administered in combination.

\section{Pharmacodynamics}

Patients treated with combination utomilumab/mogamulizumab showed a transient reduction in circulating $\mathrm{T}$ cells at $6 \mathrm{~h}(p<0.0001)$ (Fig. 1a), possibly due to cytokine-induced adhesion of $\mathrm{T}$ cells to endothelial cells
[34]. There was not a significant $(p<0.05)$ relationship between combination dose, T-cell reduction, and cytokine levels observed in this study (data on file; Pfizer). Levels of circulating $\mathrm{CD}^{+} \mathrm{T}$ cells largely returned to baseline by 7 days $(168 \mathrm{~h})$ after start of dosing; however, circulating $\mathrm{CD}^{+} \mathrm{T}$ cells did not fully recover by $336 \mathrm{~h}$. Statistically significant $(p<0.001)$ decreases in Tregs $\left(\mathrm{CD} 3+\mathrm{CD} 4+\mathrm{CD} 25+\mathrm{CD} 127^{\text {low/- }}\right)$ were observed at 6 , 168, and $336 \mathrm{~h}$ (Fig. 1b), as predicted based on data from a phase I study of single-agent mogamulizumab in patients with CCR4 $4^{-}$lung and esophageal cancers [25]. Reductions in effector memory $\left(\mathrm{CD} 45 \mathrm{RA}^{-} \mathrm{CCR} 7^{-}\right)$and central memory $\left(\mathrm{CD} 45 \mathrm{RA}^{-} \mathrm{CCR} 7^{+}\right) \mathrm{CD} 4 \mathrm{~T}$ cells were also observed at the same time points $(p<0.01)$. Naïve $\left(C D 45 \mathrm{RA}^{+} \mathrm{CCR}^{+}\right) \mathrm{CD}^{+} \mathrm{T}$ cells were less strongly affected. Within the $\mathrm{CD} 8^{+} \mathrm{T}$-cell compartment, the central memory subpopulation was reduced, relative to baseline, at 6,168 , and $336 \mathrm{~h}(p<0.005)$ to a greater degree than either the naïve or effector memory compartments (Fig. 1c). These results are largely concordant with earlier

Table 2 Descriptive summary of serum utomilumab pharmacokinetic parameter values for Cycle 5

\begin{tabular}{|c|c|c|c|}
\hline \multirow[t]{2}{*}{ Parameter, Units } & \multicolumn{3}{|c|}{ Mogamulizumab 1 mg/kg + Utomilumab, by Dose Group ${ }^{\text {a }}$} \\
\hline & $1.2 \mathrm{mg} / \mathrm{kg}$ & $100 \mathrm{mg}$ & $5 \mathrm{mg} / \mathrm{kg}$ \\
\hline$n$ & 2 & 1 & 2 \\
\hline$A \cup C_{\text {last }}, \mu \mathrm{g} \cdot \mathrm{h} / \mathrm{mL}$ & 907,1440 & 2700 & 1620,9270 \\
\hline$A \cup C_{\text {last }}(\mathrm{dn}), \mu \mathrm{g} \cdot \mathrm{h} / \mathrm{mL} / \mathrm{mg} / \mathrm{kg}$ & 756,1200 & 2490 & 323,1850 \\
\hline$C_{\max }, \mu g / m L$ & $17.5,20.0$ & 27.8 & $86.6,129$ \\
\hline$C_{\max }(\mathrm{dn}), \mu \mathrm{g} / \mathrm{mL} / \mathrm{mg} / \mathrm{kg}$ & $14.6,16.7$ & 25.6 & $17.3,25.8$ \\
\hline$C_{\text {trough, }} \mu \mathrm{g} / \mathrm{mL}$ & $1.30^{\mathrm{b}}$ & 1.16 & $2.19,5.48$ \\
\hline $\mathrm{T}_{\text {last }}, \mathrm{h}$ & 335,336 & 309 & $25.1,170$ \\
\hline$T_{\max } h$ & $2.00,2.03$ & 6.00 & $1.00,1.15$ \\
\hline
\end{tabular}

andividual patient value(s) are presented when $N<3$

${ }^{\mathrm{b}}$ Only 1 patient had quantifiable $C_{\text {trough }}$ concentrations

$A \cup C_{\text {last }}$ Area under the serum concentration-time profile from time zero to the time of the last quantifiable concentration, $C_{\max }$ Maximum observed serum concentration, $C_{\text {trough }}$ Predose concentration during multiple dosing, $d n$ Dose normalized, $n$ Number of patients in the treatment group and contributing to the summary statistics, $T_{\text {last }}$ Time of last measurable concentration, $T_{\max }$ Time for $C_{\max }$ 

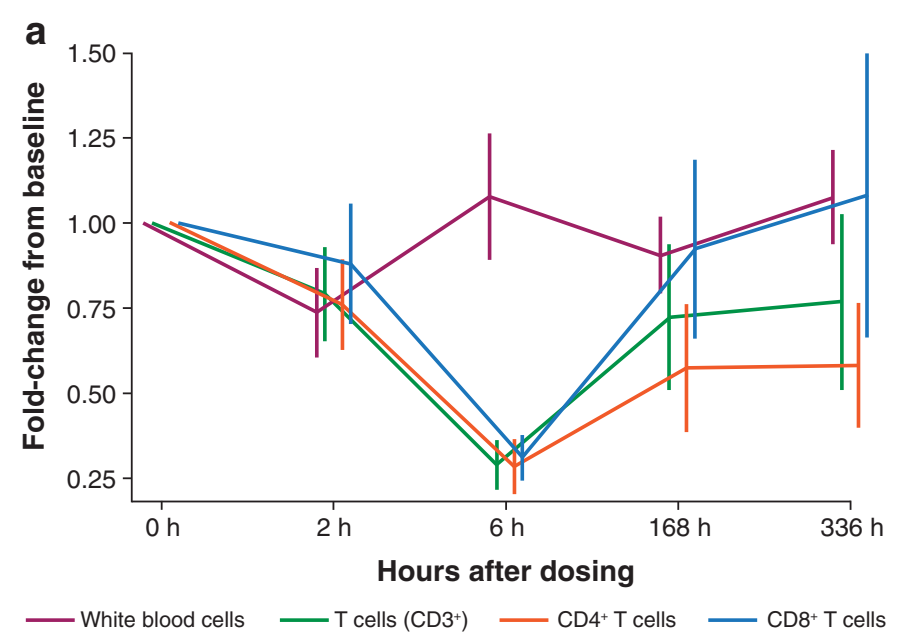

b

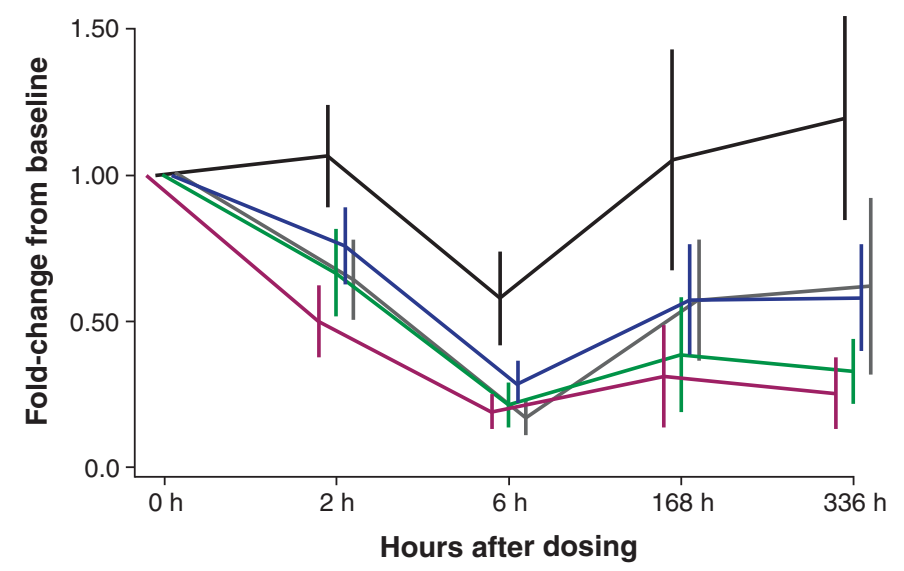

Treg Naive CD $4^{+}-$Central memory CD4 ${ }^{+}$

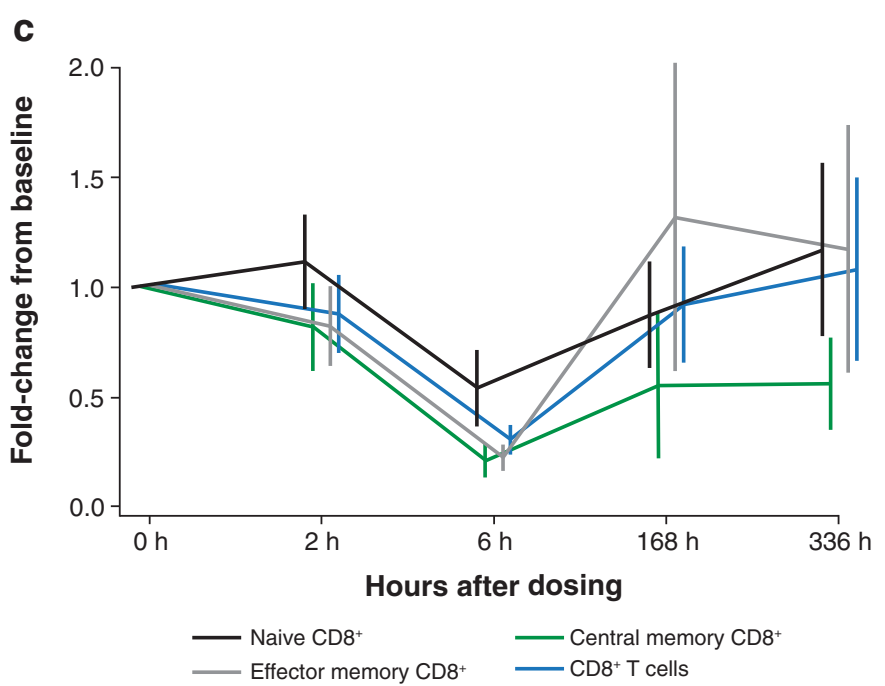

Fig. 1 (See legend on next page.) 
(See figure on previous page.)

Fig. 1 Fold-changes relative to baseline. Fold-changes are shown by lymphocyte populations in peripheral blood following treatment with utomilumab and mogamulizumab. Results were aggregated across all utomilumab doses, as statistically significant differences between utomilumab doses were not observed. a Major T-cell subpopulations relative to all white blood cells; $\mathbf{b}$ Treg and other major CD4 ${ }^{+} \mathrm{T}$ - cell populations; and (c) major CD8 ${ }^{+}$T-cell populations. White blood cells were defined by forward and side light scatter. T cells were defined by co-expression of CD3, CD4, and CD8. Naïve, central memory, and effector memory T-cell subpopulations were defined as CD45RA ${ }^{+} C C R 7^{+}$, $\mathrm{CD}_{4} \mathrm{RA}^{-} \mathrm{CCR7} 7^{+}$, and $\mathrm{CD} 45 \mathrm{RA}^{-} \mathrm{CCR7} 7^{-}$, respectively [32]. Tregs were defined as $\mathrm{CD}^{+} \mathrm{CD} 4^{+} \mathrm{CD} 25^{+} \mathrm{CD} 127^{\text {low/ }}-[33]$. Treg, regulatory T cell

findings [25] and consistent with observations that CCR4 is expressed by central memory T cells $[35,36]$.

Longitudinal analysis of TCR $\beta$ CDR3 sequences in peripheral blood by immunosequencing has been used to track individual responses to a yellow fever vaccine without prior knowledge of antigen specificity [37]. Immunosequencing was performed on peripheral blood specimens from study patients, and expanded TCR $\beta$ CDR3 sequences were identified using a beta binomial model that controls for normal biologic variance over time [28]. Comparison of the number of expanded clones in patients treated with combination utomilu$\mathrm{mab} /$ mogamulizumab to the number of expanded clones in patients treated with single-agent utomilumab [22] suggests that the addition of mogamulizumab could promote peripheral T-cell expansion ( $p<0.001)$, (Fig. 2).

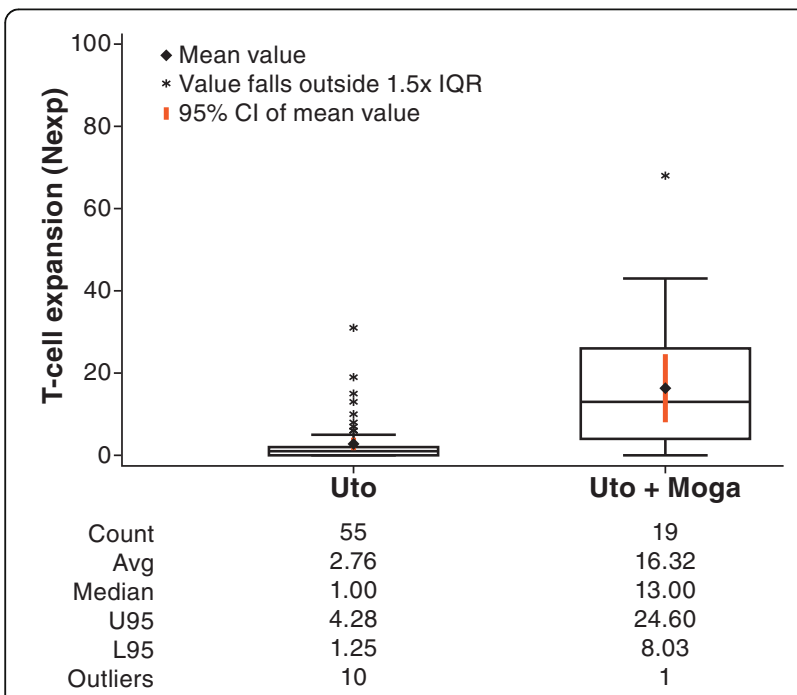

Fig. 2 TCR expansion in peripheral blood in a cohort of patients treated for 1 cycle (4 weeks) with utomilumab single-agent [22] vs patients treated with utomilumab/mogamulizumab. Individual TCR sequences were considered to have expanded after treatment if frequencies in on-treatment specimens were greater than normal biologic time-dependent variance as determined by a beta binomial model [28]. Box plot provides median and 25\%/75\% quartiles with whiskers to the last data point within $1.5 \times$ the IQR. Cl, confidence interval; IQR, interquartile range; Moga, mogamulizumab; TCR, T-cell receptor; Uto, utomilumab

\section{Characterization of baseline tumor biopsies}

Baseline tumor biopsies from patients with NSCLC $(n=1)$ and SCCHN $(n=4)$ were analyzed for the presence of PD-L1, CD8, FoxP3, and 4-1BB/CD137 (Table 3). All biopsies were negative for PD-L1 expression on tumor cells, except for one SCCHN specimen that had $10 \%$ PD-L1 ${ }^{+}$tumor cells. The biopsies had low levels of infiltrating $\mathrm{CD}^{+}$(range, 1-17\%) and FoxP3 ${ }^{+}$ (range, 1-9\%) cells, with $\mathrm{CD} 8 / \mathrm{FoxP} 3$ ratios ranging from 1 to 9 . Also, 4-1BB/CD137 was observed on small percentages $(9,10$, and $14 \%)$ of cells in the IM. The limited number of available specimens precludes generalizations about the cohort.

\section{Efficacy}

The ORR was 4.2\% (95\% CI: 0.1-21.1\%). Best percent change from baseline in sum of longest diameters (SLD) for target lesions is shown in the waterfall plot (Fig. 3a). The spider plot (Fig. 3b) shows percentage change from baseline in sum of SLD for target lesions over time. One patient in the utomilumab $100 \mathrm{mg} /$ mogamulizumab $1 \mathrm{mg} / \mathrm{kg}$ group with $\mathrm{PD}-1$ refractory squamous NSCLC achieved PR, which occurred at the first tumor assessment with a duration of response of approximately 2 months.

The patient with PR had previously received carboplatin and paclitaxel as first-line therapy (BOR of PR) and nivolumab as second-line therapy (BOR of progressive disease), and prior brain radiotherapy. This patient tested positive for both ADA and NAb against utomiliumab. The tumor was negative for PD-L1, with low levels of infiltrating $\mathrm{T}$ lymphocytes while demonstrating relatively higher numbers of $4-1 \mathrm{BB} / \mathrm{CD} 137^{+}$cells and an elevated CD8/FoxP3 ratio (Table 3). No patients achieved a BOR of CR. Nine patients had BOR of SD, 10 patients had BOR of progressive disease, and 4 were not evaluable. Representative baseline and post-treatment scans highlighting tumor shrinkage in the patient achieving PR are shown in Fig. 3c.

\section{Discussion}

In this phase I study of the combination of utomilumab with mogamulizumab in patients with advanced solid tumors, the MTD for utomilumab was determined to be at least $2.4 \mathrm{mg} / \mathrm{kg}$, and utomilumab doses up to $5 \mathrm{mg} / \mathrm{kg}$ combined with mogamulizumab $1 \mathrm{mg} / \mathrm{kg}$ were well 
Table 3 Immunohistochemistry analysis of whole tumor and IM of pretreatment tumor biopsies

\begin{tabular}{|c|c|c|c|c|c|c|c|c|c|}
\hline Cancer Type & BOR & \%PD-L1 ${ }^{+}$ & $\% \mathrm{CD}^{+} \mathrm{ALL}^{\mathrm{a}}$ & $\% F_{0 x P 3}{ }^{+} \mathrm{ALL}^{\mathrm{a}}$ & CD8/FoxP3 ALL ${ }^{a}$ & $\% \mathrm{CB}^{+} \mathrm{IM}^{\mathrm{b}}$ & \%FoxP3 ${ }^{+} \mathrm{IM}^{\mathrm{b}}$ & $\% \mathrm{CD}_{137^{+}} \mathrm{IM}^{\mathrm{b}}$ & CD8/FoxP3 IM ${ }^{b}$ \\
\hline $\mathrm{NSCLC}$ & $P R$ & 0.00 & 1.70 & 1.72 & 0.99 & 9.96 & 1.45 & 14.21 & 6.87 \\
\hline SCCHN & PD & 0.00 & 6.89 & 5.38 & 1.28 & 13.28 & 6.48 & 8.59 & 2.05 \\
\hline $\mathrm{SCCHN}$ & SD & 0.00 & 17.16 & 1.74 & 9.86 & NE & $\mathrm{NE}$ & $N E$ & $\mathrm{NE}$ \\
\hline $\mathrm{SCCHN}$ & SD & 10.00 & 13.69 & 4.98 & 2.75 & NE & $\mathrm{NE}$ & $\mathrm{NE}$ & $\mathrm{NE}$ \\
\hline $\mathrm{SCCHN}$ & SD & 0.00 & 12.23 & 9.43 & 1.3 & 14.68 & 10.05 & 10.37 & 1.46 \\
\hline
\end{tabular}

Marker-positive cells are reported as a percent of evaluated cells

${ }^{a} A L L$ : The region encompassing the tumor and extending up to the leading edge, but not outside the tumor-normal interface

${ }^{\mathrm{b}} \mathrm{MM}$ : The region extending from $500 \mu \mathrm{m}$ outside the leading edge of the tumor to $500 \mu \mathrm{m}$ inside

$B O R$ Best overall response, NE Not evaluable, NSCLC Non-small-cell lung cancer, $P D$ Progressive disease, $P D-L 1$ Programmed cell death-ligand 1, $P R$ Partial response, SCCHN Squamous cell cancer of head and neck, SD Stable disease

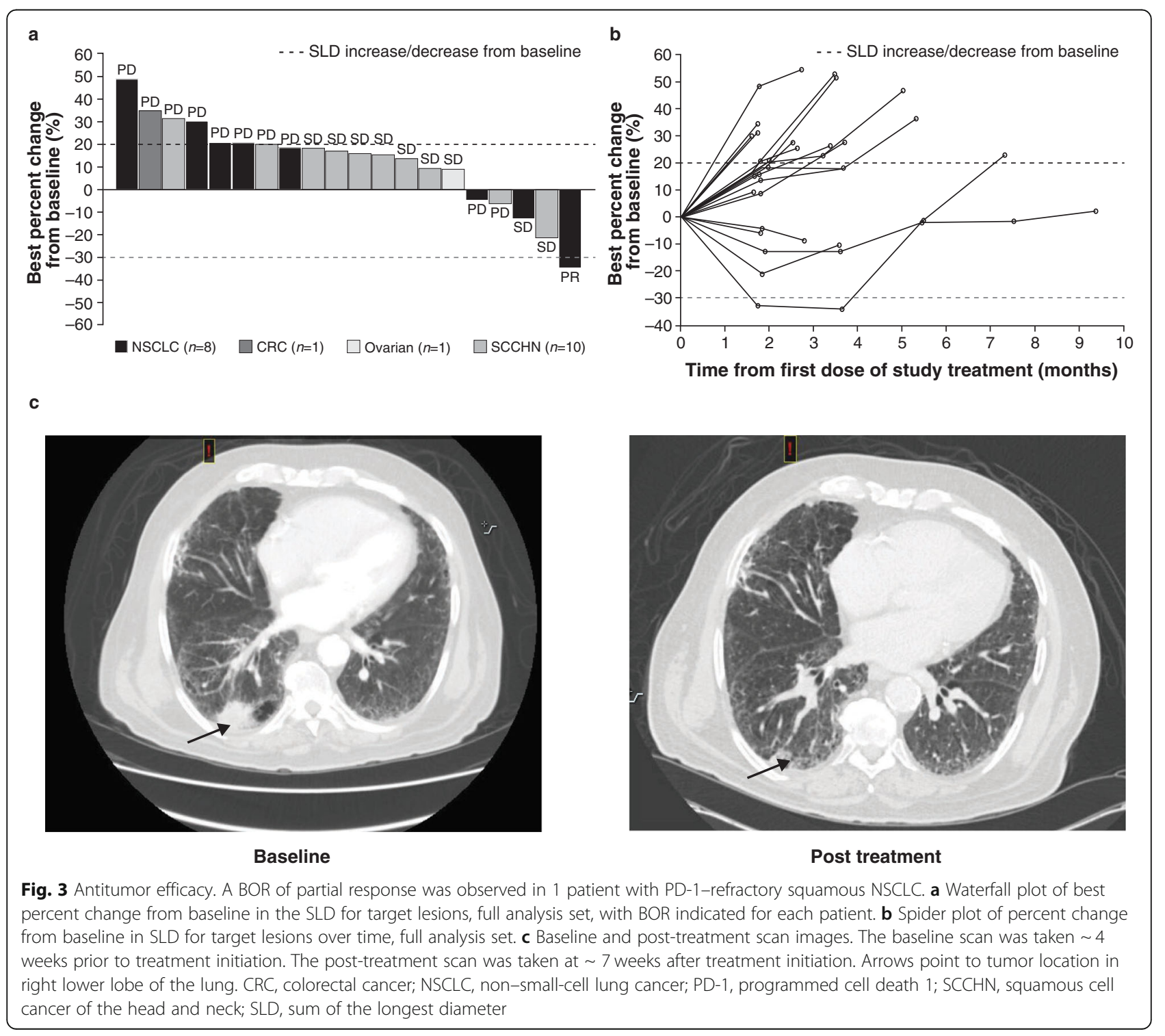


tolerated. None of the patients experienced a DLT with any dose combination. Utomilumab systemic exposure appeared to increase with each dose escalation, but the relationship between PK and dose could not be fully evaluated owing to low patient numbers. Following co-administration with utomilumab, mogamulizumab PK was similar across dose groups, with accumulation observed following multiple-dose administration. There was no substantial impact of $\mathrm{ADA} / \mathrm{NAb}$ on PK and safety.

The peripheral blood biomarker analyses performed in this study indicated that Tregs and at least some central memory $\mathrm{T}$ cells were depleted, as was observed by Kurose et al. in patients treated with single-agent mogamulizumab [25]. Expansion of TCR $\beta$ CDR3 regions in the combination cohort is consistent with the hypothesis that mogamulizumab-mediated depletion of Tregs and other $\mathrm{CCR}^{+}$cells can promote peripheral T-cell expansion, although the durability of such expansion in conjunction with potential central memory depletion cannot be assessed.

Pretreatment tumor biopsy results were only available for 5 of the 24 enrolled patients. Four tumor biopsies were PD-L1-negative, including the biopsy from the patient with NSCLC who achieved PR. One patient with SCCHN who achieved BOR of SD had a biopsy with a $10 \%$ PD-L1 tumor proportion score. It is possible that many, if not most, of the enrolled patients had tumors with minimal antitumor immune activity. The efficacy of Treg depletion in such tumors is likely contingent on the relationship between Tregs and that phenotype: if Tregs are the primary causal agent, then removing them should increase immune activity, but not if immune activity is reduced for other reasons. The two hypotheses cannot be differentiated in this study. The patient with NSCLC who achieved PR had the highest CD8/FoxP3 ratio and proportion of $4-1 \mathrm{BB} / \mathrm{CD} 137^{+}$cells in the IM, coupled with the lowest CD8/FoxP3 ratio throughout the tumor itself. This phenotype may suggest the existence of a utomilumab-responsive tumor-infiltrating lymphocyte population in the IM that is being quenched by Tregs closer to the tumor center. A larger, prospectively designed study would be required for further definition of this phenotype and estimation of its prevalence.

This study was designed to test the hypothesis that depletion of $\mathrm{CCR}^{+}{ }^{+}$Tregs would enhance the effect of anti-tumor $\mathrm{T}$ cells expanded in response to a $4-1 \mathrm{BB}$ agonist. While the observed depletion of Tregs coupled with TCR expansion in the peripheral blood is consistent with this hypothesis, it is possible that other effects of CCR4 depletion may affect clinical outcome. For instance, depletion of CCR4 ${ }^{+} \mathrm{T}$ cell types, such as memory, Th1, Th2, and resident memory $\mathrm{T}$ cells [38-40] could impact the anti-tumor response elicited by combination therapy. It has been reported that CCR4 is required for optimal $\mathrm{T}$ cell-mediated protection from influenza in mice [41], and surface CCR4 expression has been observed on lymphocytes isolated from lung and bronchoalveolar lavage fluid [42]. The transience of the PR seen in a PD-1 refractory squamous NSCLC patient may be consistent with attenuation of anti-tumor activity mediated by CCR4-expressing $\mathrm{T}$ cells. It is also possible that depletion of CCR4-expressing $\mathrm{T}$ cells leaves other tumor-infiltrating Tregs unaffected, such as the CCR8expressing Tregs that have been noted in multiple tumor types $[43,44]$.

\section{Conclusion}

The combination of utomilumab plus mogamulizumab was well tolerated in patients with advanced solid tumors, with a PR achieved by 1 NSCLC patient. The results of the translational analyses are consistent with the hypothesized mechanism of action. Clinical benefit from this combination may be meaningful for patients in whom CCR4+ Tregs have induced a dormant $\mathrm{CD} 8^{\text {low }} /$ $\mathrm{PD}-\mathrm{L} 1^{\text {low }}$ phenotype that may be unresponsive to antiPD-1/PD-L1 therapy.

\section{Supplementary information}

Supplementary information accompanies this paper at https://doi.org/10. 1186/s40425-019-0815-6.

Additional file 1. Descriptive summary of serum mogamulizumab pharmacokinetic parameter values for Cycle 1, single dose. Table of PK values after one dose.

Additional file 2. Descriptive summary of serum mogamulizumab pharmacokinetic parameter values for Cycle 5 (biweekly dosing). Table of PK values after multiple dosing.

\section{Abbreviations}

ADA: Antidrug antibody; AE: Adverse event; ATL: Adult-cell leukemialymphoma; BOR: Best overall response; CCR4: C-C chemokine receptor 4; $\mathrm{Cl}$ : Confidence interval; CR: Complete response; CRC: Colorectal cancer; DLT: Dose-limiting toxicity; ECL: Electrochemiluminescence; EOT: End of treatment; IM: Invasive margin; IQR: Interquartile range; KHK: Kyowa Hakko Kirin; mAB: Monoclonal antibody; Moga: Mogamulizumab; MTD: Maximum tolerated dose; NAb: Neutralizing antibody; NSCLC: Non-small-cell lung cancer; ORR: Objective response rate; PD: Progressive disease; PD-1: Programmed cell death 1; PD-L1: Programmed cell death-ligand 1; PK: Pharmacokinetics; PR: Partial response; r/r: Relapse/refractory; SCCHN: Squamous cell carcinoma of head and neck; SD: Stable disease; SLD: Sum of the longest diameter; TCR: T-cell receptor; TITE-CRM: Time-to-event continual reassessment method; Treg: Regulated T cell; Uto: Utomilumab

\section{Acknowledgements}

We acknowledge Chetan Deshpande, Ren-Yu Tzeng, and Shibing Deng for their assistance with the biomarker analyses. Medical writing support was provided by David Sunter, PhD, CMPP, of Engage Scientific Solutions and was funded by Pfizer Inc.

Authors' contributions

All authors participated in the interpretation of study results, and in the drafting, critical revision, and approval of the final version of the manuscript. 


\section{Funding}

The design of the study and collection and analysis of data was funded by Pfizer Inc. Medical writing support was provided by David Sunter, PhD, CMPP, of Engage Scientific Solutions and was funded by Pfizer Inc.

\section{Availability of data and materials}

Upon request, and subject to certain criteria, conditions and exceptions (see https://www.pfizer.com/science/clinical-trials/trial-data-and-results for more information), Pfizer will provide access to individual de-identified participant data from Pfizer-sponsored global interventional clinical studies conducted for medicines, vaccines and medical devices (1) for indications that have been approved in the US and/or EU or (2) in programs that have been terminated (i.e., development for all indications has been discontinued). Pfizer will also consider requests for the protocol, data dictionary, and statistical analysis plan. Data may be requested from Pfizer trials 24 months after study completion. The de-identified participant data will be made available to researchers whose proposals meet the research criteria and other conditions, and for which an exception does not apply, via a secure portal. To gain access, data requestors must enter into a data access agreement with Pfizer.

\section{Ethics approval and consent to participate}

The study was conducted in compliance with the ethical principles originating in or derived from the Declaration of Helsinki and in compliance with the International Council for Harmonisation Good Clinical Practice Guidelines. All patients provided written informed consent. The final protocol, any amendments and informed consent documentations were reviewed and approved by the Institutional Review Boards at each of the investigational centers participating in the study.

\section{Consent for publication}

Not applicable.

\section{Competing interests}

EC has received funding from AstraZeneca, Amgen, Bayer, Bristol-Myers Squibb, MSD, and Merck; MP has no conflicts of interest. DS has no conflicts of interest that directly affect this trial, has received research funding from Pfizer, BMS, Celgene, Leap, and Alkermes; is consultant for Sanofi/Genzyme, Aduro, Eli Lilly, AstraZeneca, and Ipsen; and is on the speakers' bureau for Eli Lilly, Genentech, and Celgene; MLJ has received research funding from BerGenBio, Eli Lilly, EMD Serono, Janssen, Mirati Therapeutics, Genmab, Pfizer, AstraZeneca, Genentech/Roche, Stemcentrix, Novartis, Checkpoint Therapeutics, Array Biopharma, Regeneron, Apexigen, AbbVie, Tarveda, Adaptimmune, Syndax, Neovia, Boehringer Ingelheim, Sanofi, Hengrui Therapeutics, INC, Merck, Daiichi-Sankyo, Lycera, G1 Therapeutics, Dynavax, LOXO, Cytomx, BeiGene, Birdie, Corvus, Incyte, Genocea, Gritstone, Amgen, Bristol-Myers Squibb, Kadmon, Clovis, Acerta, OncoMed, and Guardant Health; and has served as a consultant for Genentech/Roche, Celgene, Boehringer Ingelheim, Sanofi, Mirati Therapeutics, LOXO, Calithera, AstraZeneca, Merck, Araxes Pharma, Mersana Therapeutics, BeiGene, Incyte, Pfizer, Guardant Health, Bristol-Myers Squibb, and Ribon Therapeutics. CC has received honoraria for advisory boards with Bristol-Myers Squibb, AstraZeneca, Celgene, Ignyta, CUE Biopharma, and Eli Lilly. SP has received research funding from Merck, Pfizer, Bristol-Myers Squibb, Vyriad, Genentech, and Novartis and has served as a consultant for Bristol-Myers Squibb. DW has no conflicts of interest. JW has received research funding from AstraZeneca, Merck, Pfizer, Celgene, Novartis, and G1 and is consultant and served on advisory panels for AstraZeneca, Pfizer, Nanobiotox, G1, and Immunicum. YC, CBD, BH, FT, and AT are employees of Pfizer and hold stock in Pfizer.

\section{Author details}

${ }^{1}$ UC San Diego Health, Moores Cancer Center, University of California San Diego, 3855 Health Sciences Drive, La Jolla, CA 92093, USA. ²Georgetown University Medical Center, Washington, DC, USA. ${ }^{3}$ Cleveland Clinic, Cleveland, $\mathrm{OH}$, USA. ${ }^{4}$ Henry Ford Hospital, Detroit, MI, USA. ${ }^{5}$ University of North Carolina at Chapel Hill, Chapel Hill, NC, USA. ${ }^{6}$ Sarah Cannon Research Institute, Nashville, TN, USA. ' Moffit Cancer Center, Tampa, FL, USA. ${ }^{8}$ Pfizer Inc, La Jolla, CA, USA. ${ }^{9}$ Pfizer Inc, New York, NY, USA. ${ }^{10}$ Pfizer Inc, Milan, Italy. ${ }^{11}$ Sanford Research, Sioux Falls, SD, USA.
Received: 6 September 2019 Accepted: 11 November 2019 Published online: 04 December 2019

\section{References}

1. Aguiar PN, De Mello RA, Barreto CMN, Perry LA, Penny-Dimri J, Tadokoro H, et al. Immune checkpoint inhibitors for advanced non-small cell lung cancer: emerging sequencing for new treatment targets. ESMO Open. 2017; 2:e000200.

2. Forster MD, Devlin M-J. Immune checkpoint inhibition in head and neck cancer. Front Oncol. 2018;8:310-9.

3. Karlsson AK, Saleh SN. Checkpoint inhibitors for malignant melanoma: a systematic review and meta-analysis. Clin Cosmet Investig Dermatol. 2017;10:325-39.

4. Ghatalia P, Zibelman M, Geynisman DM, Plimack E. Approved checkpoint inhibitors in bladder cancer: which drug should be used when? Ther Adv Med Oncol. 2018:10:1-10.

5. Yip SM, Wells C, Moreira R, Wong A, Srinivas S, Beuselinck B, et al. Checkpoint inhibitors in patients with metastatic renal cell carcinoma: results from the international metastatic renal cell carcinoma database consortium. Cancer. 2018;124:3677-83.

6. Dougan M, Dranoff G, Dougan SK. Cancer immunotherapy: beyond checkpoint blockade. Ann Rev Cancer Biol. 2019;3:55-75.

7. Sharma P, Hu-Lieskovan S, Wargo JA, Ribas A. Primary, adaptive, and acquired resistance to cancer immunotherapy. Cell. 2017;168:707-23.

8. Fisher TS, Kamperschroer C, Oliphant T, Love VA, Lira PD, Doyonnas R, et al. Targeting of 4-1BB by monoclonal antibody PF-05082566 enhances T-cell function and promotes anti-tumor activity. Cancer Immunol Immunother. 2012;61:1721-33.

9. Vinay DS, Kwon BS. 4-1BB (CD137), an inducible costimulatory receptor, as a specific target for cancer therapy. BMB Rep. 2014;47:122-9.

10. Choi BK, Kim YH, Kwon PM, Lee SC, Kang SW, Kim MS, et al. 4-1BB functions as a survival factor in dendritic cells. J Immunol. 2009;182:4107-15.

11. Marvel J, Walzer T. CD137 in NK cells. Blood. 2010;115:2987.

12. Lynch $\mathrm{DH}$. The promise of 4-1BB (CD137)-mediated immunomodulation and the immunotherapy of cancer. Immunol Rev. 2008;222:277-86.

13. Houot R, Goldstein MJ, Kohrt HE, Myklebust JH, Alizadeh AA, Lin JT, et al. Therapeutic effect of CD137 immunomodulation in lymphoma and its enhancement by Treg depletion. Blood. 2009:114:3431-8.

14. Sanchez-Paulete AR, Labiano S, Rodriguez-Ruiz ME, Azpilikueta A, Etxeberria I, Bolanos E, et al. Deciphering CD137 (4-1BB) signaling in T-cell costimulation for translation into successful cancer immunotherapy. Eur J Immunol. 2016:46:513-22.

15. Vinay DS, Kwon BS. Therapeutic potential of anti-CD137 (4-1BB) monoclonal antibodies. Expert Opin Ther Targets. 2016;20:361-73.

16. Takahashi C, Mittler RS, Vella AT. Cutting edge: 4-1BB is a bona fide CD8 T cell survival signal. J Immunol. 1999;162:5037.

17. Cannons JL, Lau P, Ghumman B, DeBenedette MA, Yagita H, Okumura K, et al. 4-1BB ligand induces cell division, sustains survival, and enhances effector function of CD4 and CD8 T cells with similar efficacy. J Immunol. 2001;167:1313.

18. Bertram EM, Lau P, Watts TH. Temporal segregation of 4-1BB versus CD28mediated costimulation: 4-1BB ligand influences $T$ cell numbers late in the primary response and regulates the size of the $T$ cell memory response following influenza infection. J Immunol. 2002;168:3777.

19. Chen S, Lee LF, Fisher TS, Jessen B, Elliott M, Evering W, et al. Combination of 4-1BB agonist and PD-1 antagonist promotes antitumor effector/memory CD8 T cells in a poorly immunogenic tumor model. Cancer Immunol Res. 2015;3:149-60.

20. Chacon JA, Wu RC, Sukhumalchandra P, Molldrem JJ, Sarnaik A, PilonThomas S, et al. Co-stimulation through 4-1BB/CD137 improves the expansion and function of CD8(+) melanoma tumor-infiltrating lymphocytes for adoptive T-cell therapy. PLoS One. 2013;8:e60031.

21. Srivastava RM, Trivedi S, Concha-Benavente F, Gibson SP, Reeder C, Ferrone S, et al. CD137 stimulation enhances cetuximab-induced natural killer: dendritic cell priming of antitumor T-cell immunity in patients with head and neck cancer. Clin Cancer Res. 2017;23:707-16.

22. Segal NH, He AR, Doi T, Levy R, Bhatia S, Pishvaian MJ, et al. Phase I study of single-agent utomilumab (PF-05082566), a 4-1BB/CD137 agonist, in patients with advanced cancer. Clin Cancer Res. 2018:24:1816-23.

23. Yuan Q, Bromley SK, Means TK, Jones K, Hayashi F, Bhan AK, et al. CCR4dependent regulatory T cell function in inflammatory bowel disease. J Exp Med. 2007;204:1327-34. 
24. Sugiyama D, Nishikawa H, Maeda Y, Nishioka M, Tanemura A, Katayama I, et al. Anti-CCR4 mAb selectively depletes effector-type FoxP3(+)CD4(+) regulatory T cells, evoking antitumor immune responses in humans. Proc Natl Acad Sci U S A. 2013;110:17945-50

25. Kurose K, Ohue Y, Wada H, lida S, Ishida T, Kojima T, et al. Phase la study of FoxP3+ CD4 Treg depletion by infusion of a humanized anti-CCR4 antibody, KW-0761, in cancer patients. Clin Cancer Res. 2015;21:4327-36.

26. Choi BK, Kim YH, Kang WJ, Lee SK, Kim KH, Shin SM, et al. Mechanisms involved in synergistic anticancer immunity of anti-4-1BB and anti-CD4 therapy. Cancer Res. 2007;67:8891-9.

27. Tolcher AW, Sznol M, Hu-Lieskovan S, Papadopoulos KP, Patnaik A, Rasco D, et al. Phase lb study of utomilumab (PF-05082566), a 4-1BB/CD137 agonist, in combination with pembrolizumab (MK-3475) in patients with advanced solid tumors. Clin Cancer Res. 2017;23:5349-57.

28. Rytlewski J, Deng S, Xie T, Davis C, Robins H, Yusko E, et al. Model to improve specificity for identification of clinically-relevant expanded $T$ cells in peripheral blood. PLoS One. 2019;14:e0213684.

29. Cheung YK, Chappell R. Sequential designs for phase I clinical trials with late-onset toxicities. Biometrics. 2000;56:1177-82.

30. Huang B, Kuan PF. Time-to-event continual reassessment method incorporating treatment cycle information with application to an oncology phase I trial. Biom J. 2014;56:933-46

31. Huang B, Bycott P, Talukder E. Novel dose-finding designs and considerations on practical implementations in oncology clinical trials. J Biopharm Stat. 2017;27:44-55.

32. Sallusto F, Lenig D, Förster R, Lipp M, Lanzavecchia A. Two subsets of memory T lymphocytes with distinct homing potentials and effector functions. Nature. 1999:401:708-12.

33. Yu N, Li X, Song W, Li D, Yu D, Zeng X, et al. CD4+CD25+CD127low/- T cells: a more specific Treg population in human peripheral blood. Inflammation. 2012;35:1773-80.

34. Watson C, Whittaker S, Smith N, Vora AJ, Dumonde DC, Brown KA. IL-6 acts on endothelial cells to preferentially increase their adherence for lymphocytes. Clin Exp Immunol. 1996;105:112-9.

35. Kondo T, Takiguchi M. Human memory CCR4+CD8+ T cell subset has the ability to produce multiple cytokines. Int Immunol. 2009;21:523-32.

36. Rivino L, Messi M, Jarrossay D, Lanzavecchia A, Sallusto F, Geginat J. Chemokine receptor expression identifies pre-T helper (Th)1, pre-Th2, and nonpolarized cells among human CD4+ central memory T cells. J Exp Med. 2004;200:725-35.

37. DeWitt WS, Emerson RO, Lindau P, Vignali M, Snyder TM, Desmarais C, et al. Dynamics of the cytotoxic $T$ cell response to a model of acute viral infection. J Virol. 2015;89:4517.

38. Andrew DP, Ruffing N, Kim CH, Miao W, Heath H, Li Y, et al. C-C chemokine receptor 4 expression defines a major subset of circulating nonintestinal memory T cells of both Th1 and Th2 potential. J Immunol. 2001;166:103-11.

39. lellem A, Mariani M, Lang R, Recalde H, Panina-Bordignon P, Sinigaglia F, et al. Unique chemotactic response profile and specific expression of chemokine receptors CCR4 and CCR8 by CD4(+)CD25(+) regulatory T cells. J Exp Med. 2001:194:847-53.

40. Clark RA. Resident memory T cells in human health and disease. Sci Transl Med. 2015;7:269rv1.

41. Mikhak Z, Strassner JP, Luster AD. Lung dendritic cells imprint T cell lung homing and promote lung immunity through the chemokine receptor CCR4. J Exp Med. 2013;210:1855-69.

42. Kunkel EJ, Boisvert J, Murphy K, Vierra MA, Genovese MC, Wardlaw AJ, et al. Expression of the chemokine receptors CCR4, CCR5, and CXCR3 by human tissue-infiltrating lymphocytes. Am J Pathol. 2002;160:347-55.

43. De Simone M, Arrigoni A, Rossetti G, Gruarin P, Ranzani V, Politano C, et al. Transcriptional landscape of human tissue lymphocytes unveils uniqueness of tumor-infiltrating T regulatory cells. Immunity. 2016:45:1135-47.

44. Plitas G, Konopacki C, Wu K, Bos PD, Morrow M, Putintseva EV, et al. Regulatory $T$ cells exhibit distinct features in human breast Cancer. Immunity. 2016;45:1122-34.

\section{Publisher's Note}

Springer Nature remains neutral with regard to jurisdictional claims in published maps and institutional affiliations.

\section{Ready to submit your research? Choose BMC and benefit from:}

- fast, convenient online submission

- thorough peer review by experienced researchers in your field

- rapid publication on acceptance

- support for research data, including large and complex data types

- gold Open Access which fosters wider collaboration and increased citations

- maximum visibility for your research: over $100 \mathrm{M}$ website views per year

At $\mathrm{BMC}$, research is always in progress.

Learn more biomedcentral.com/submissions 